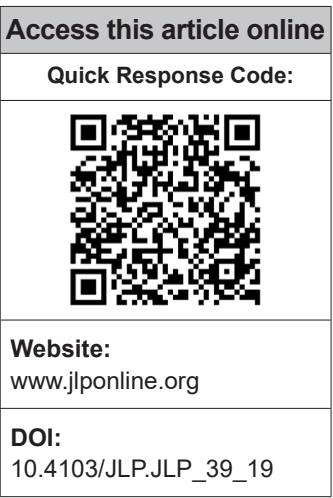

DOI:

/JLP.JLP 39
Department of Microbiology, All India Institute of Medical Sciences, Bhubaneswar,

Odisha, India

Address for correspondence: Dr. Srujana Mohanty,

Department of Microbiology, All India Institute of Medical Sciences, Bhubaneswar - 751019 , Odisha, India E-mail: srujana_micro@ yahoo.co.in

Submission: 03-03-2019 Accepted: 09-09-2019

\section{Recent pattern of antibiotic resistance in Staphylococcus aureus clinical isolates in Eastern India and the emergence of reduced susceptibility to vancomycin}

\author{
Srujana Mohanty, Bijayini Behera, Subhrajyoti Sahu, Ashok Kumar Praharaj
}

\begin{abstract}
:
PURPOSE: We aimed to determine the recent pattern of antibiotic resistance and assess the vancomycin susceptibility profile of clinical Staphylococcus aureus in view of emerging reports of vancomycin creep, reduced vancomycin susceptibility (RVS), including heterogeneous vancomycin-intermediate $S$. aureus (hVISA) and vancomycin-intermediate $S$. aureus, and vancomycin resistance in $S$. aureus isolates.
\end{abstract}

MATERIALS AND METHODS: Consecutive, nonduplicate isolates of S. aureus between July 2015 and June 2016 were subjected to antimicrobial susceptibility testing using standard disk diffusion test or Etest as per the Clinical and Laboratory Standards Institute 2015. Detection of hVISA was done by glycopeptide resistance detection Etest according to the manufacturer's instructions in strains with vancomycin minimum inhibitory concentration of $1-2 \mu \mathrm{g} / \mathrm{ml}$.

RESULTS: A total of 284 S. aureus were obtained from pus $(175,61.6 \%)$, respiratory tract $(31,10.9 \%)$, urine $(27,9.5 \%)$, blood $(25,8.8 \%)$, body fluids $(18,6.3 \%)$, and catheter tips $(8,2.8 \%) .127(44.7 \%)$ isolates were methicillin resistant, and 158 (55.6\%) were multidrug resistant. High resistance was observed to penicillin $(81.7 \%)$, erythromycin $(62.3 \%)$, and ciprofloxacin $(52.1 \%)$, whereas the resistance was low to gentamicin (5.3\%), rifampicin (8.1\%), and doxycycline (9.5\%). Two hundred and fifty-one $(88.3 \%)$ isolates were fully susceptible to vancomycin, whereas $33(11.6 \%)$ demonstrated RVS. All were uniformly susceptible to linezolid, tigecycline, and daptomycin.

CONCLUSIONS: A moderately high percentage of $S$. aureus isolates demonstrated RVS, which may limit its usefulness in methicillin-resistant isolates and may be associated with increased complications in methicillin-susceptible infections. In view of increasing glycopeptide resistance, the susceptibility status of vancomycin along with other antibiotics among clinical $\mathrm{S}$. aureus isolates should be investigated periodically.

Keywords:

Heterogenous vancomycin-intermediate Staphylococcus aureus, methicillin-resistant Staphylococcus aureus, Staphylococcus aureus, vancomycin, vancomycin-intermediate Staphylococcus aureus

\section{Introduction}

Ctaphylococcus aureus is an important $\checkmark$ pathogen in both community and

This is an open access journal, and articles are distributed under the terms of the Creative Commons Attribution-NonCommercial-ShareAlike 4.0 License, which allows others to remix, tweak, and build upon the work non-commercially, as long as appropriate credit is given and the new creations are licensed under the identical terms.

For reprints contact: reprints@medknow.com hospital settings with manifestations ranging from relatively mild localized skin and skin-structure infections to life-threatening systemic infections, such as sepsis, necrotizing pneumonia, septic

How to cite this article: Mohanty S, Behera B, Sahu S, Praharaj AK. Recent pattern of antibiotic resistance in Staphylococcus aureus clinical isolates in Eastern India and the emergence of reduced susceptibility to vancomycin. J Lab Physicians 2019;11:340-5. 
arthritis, endocarditis, and osteomyelitis. ${ }^{[1,2]}$ Especially, the methicillin-resistant $S$. aureus (MRSA) constitutes one of the most serious contemporary challenges to treatment, being simultaneously resistant to several classes of drugs and being able to have a rapid global spread. ${ }^{[1,2]}$ For MRSA and multidrug-resistant (MDR) S. aureus infections, vancomycin is considered the mainstay of antimicrobial therapy and has been the treatment of choice for serious MRSA infections since 1958. ${ }^{[3]}$

However, emerging reports of vancomycin minimum inhibitory concentrations (MIC) creep, reduced vancomycin susceptibility (RVS), and even vancomycin resistance in clinical MRSA isolates from different geographical regions are disturbing, because of minimal alternative therapeutic options. ${ }^{[3-6]}$ This is because the clinical effectiveness of vancomycin may be compromised even against vancomycin-sensitive S. aureus (VSSA) strains whose MICs are within the Clinical and Laboratory Standards Institute (CLSI) susceptible range $(\leq 2 \mu \mathrm{g} / \mathrm{ml})$. Such heterogeneous vancomycin-intermediate $S$. aureus (hVISA) strains may evolve into VISA, leading to clinical treatment failure. ${ }^{[3,4]}$ Recently, it has also been observed that high vancomycin MIC in methicillin-susceptible S. aureus (MSSA) is associated with poorer outcomes and increased complications in patients with $S$. aureus bacteremia and endocarditis. ${ }^{[7,8]}$ Thus, determination of vancomycin MIC and the ability to accurately identify $S$. aureus isolates with reduced susceptibility to vancomycin is of paramount importance for several reasons such as successful clinical outcomes, the targeted treatment of MRSA bacteremia, or as a prognostic marker in MSSA bacteremia.

Because RVS in S. aureus is a complex phenomenon and difficult to detect in clinical microbiology laboratories, the CLSI in January 2006 redefined and updated the resistance breakpoints for vancomycin against $S$. aureus (susceptible, intermediate, or resistant when the vancomycin MIC is $\leq 2 \mu \mathrm{g} / \mathrm{ml}, 4-8 \mu \mathrm{g} / \mathrm{ml}$, or $\geq 16 \mu \mathrm{g} / \mathrm{ml}$, respectively) to increase the sensitivity in detecting the nonsusceptible isolates. ${ }^{[9]}$ In addition, in 2009, the CLSI altered the guidelines for staphylococci such that disk diffusion was no longer an acceptable means for testing vancomycin susceptibility in these organisms. ${ }^{[10]}$ For the detection of hVISA, though the population analysis profile-area under the curve (PAP-AUC) ratio is regarded as the gold standard test, it is relatively cumbersome, time-consuming, labor-intensive, and difficult to perform in a clinical diagnostic laboratory. Hence, newer tests such as the glycopeptide resistance detection (GRD) Etest and the macromethod Etest are being used recently. ${ }^{[11,12]}$ With the emergence of RVS, various alternative therapeutic options have been advocated for the treatment of resistant $S$. aureus infections such as ceftaroline, tigecycline, linezolid, telavancin, and daptomycin, which have shown considerable clinical efficacy against $S$. aureus in various studies. ${ }^{[13,14]}$

Only a few studies from India have previously reported reduced susceptibility to vancomycin after the introduction of the revised CLSI guidelines. ${ }^{[15-19]}$ Hence, we aimed to determine the recent pattern of antibiotic resistance, assess the vancomycin susceptibility profile, and ascertain other therapeutic options for clinical $S$. aureus isolates in Eastern India, which would help in choosing empirical antimicrobial therapy as well as in administering targeted therapy to the patients.

\section{Materials and Methods}

The study, approved by the institutional ethical committee, was conducted over a period of 1 year from July 2015 to June 2016 in a tertiary care research, referral, and teaching hospital in Eastern India. The hospital receives patients not only from the local areas but also from adjoining districts and states who are referred.

\section{Bacterial isolates}

Consecutive nonrepeat, nonduplicate $S$. aureus isolates obtained from patients with clinically diagnosed infections (one isolate per patient) whose clinical specimens were submitted to the department of microbiology by the treating physician were included in the study. The isolates were identified by colony characteristics, Gram stain reaction, and standard biochemical tests. ${ }^{[20]}$

Antimicrobial susceptibility testing, minimum inhibitory concentrations determination, and detection of heterogeneous vancomycinintermediate Staphylococcus aureus

Susceptibility testing of $S$. aureus to the primary antimicrobial agents was performed by disk diffusion testing on Mueller-Hinton agar (MHA) as per the latest CLSI guidelines. ${ }^{[21]}$ All the disks were procured from HiMedia, Mumbai, Maharashtra, India. MRSA were identified by cefoxitin $(30 \mu \mathrm{g})$ disk testing and cefoxitin MIC by Etest (HiMedia, Mumbai, Maharashtra, India) as per the CLSI 2015 (zone diameter, sensitive $\geq 22 \mathrm{~mm}$ and resistant $\leq 21 \mathrm{~mm}$; MIC, sensitive $\leq 4 \mu \mathrm{g} / \mathrm{ml}$ and resistant $\geq 8 \mu \mathrm{g} / \mathrm{ml}) \cdot{ }^{[21]}$ Inducible clindamycin resistance in isolates displaying erythromycin resistance was detected by the D-test. ${ }^{[21]}$

Susceptibility testing to vancomycin was performed to determine the MIC by both Etest (HiMedia, Mumbai, Maharashtra, India) and agar dilution with incorporation of intermediate dilutions in the agar dilution test for better correlation between the two methods of testing. In case of discrepancies between Etest and 
agar dilution, the values of Etest MICs were deemed to be final because Etest has been found to be a more sensitive method for MIC determination and considered the best technique to assess exact MIC as it allows for visualization of small colonies around the zones of inhibition. ${ }^{[22]}$ With Etest as the gold standard for susceptibility testing, detection of hVISA was done by GRD Etest on MHA with a 5\% blood agar plate according to the manufacturer's instructions (HiMedia, Mumbai, Maharashtra, India) in strains with vancomycin MIC $1-2 \mu \mathrm{g} / \mathrm{ml} .{ }^{[11,22]}$ The test isolate was considered positive for hVISA if the Etest GRD strip result was $\geq 8 \mu \mathrm{g} / \mathrm{ml}$ for vancomycin or teicoplanin. ${ }^{[11,22]}$ Isolates displaying either VISA (vancomycin MIC $>2-<16 \mu \mathrm{g} / \mathrm{ml}$ ) or hVISA (detected by GRD Etest) phenotype were designated as strains with RVS. ${ }^{[4]}$

Minimum inhibitory concentration was also determined for alternative therapeutic options such as linezolid, daptomycin, and tigecycline by Etest (HiMedia, Mumbai, Maharashtra, India) and interpreted as per the CLSI guidelines, ${ }^{[21]}$ except tigecycline (for which CLSI currently does not provide interpretative zone diameters). S. aureus strains ATCC 25923, ATCC 51299, and ATCC 43300 were used as quality control strains for disk diffusion, MIC testing, and MRSA testing, respectively. Tigecycline susceptibility was interpreted as $\geq 19 \mathrm{~mm}$ zone diameter and $\leq 0.5 \mu \mathrm{g} /$ ml MIC breakpoint as per the US Food and Drug Administration guidelines, respectively. ${ }^{[23]}$ Strains with intermediate resistance were included in the percentage of resistant isolates. Multidrug resistance was defined as nonsusceptibility to at least one agent in three or more antimicrobial categories. ${ }^{[24]}$

\section{Results}

A total of 284 S. aureus were isolated during the study period with sample-wise distribution as follows: pus $(175,61.6 \%)$, respiratory samples $(31,10.9 \%)$, urine $(27,9.5 \%)$, blood $(25,8.8 \%)$, body fluids $(18,6.3 \%)$, and catheter tips $(8,2.8 \%)$. "Seventy-nine (27.8\%) isolates were from the outpatient department (OPD), whereas $205(72.2 \%)$ were from admitted patients. Of the 284 isolates, 87 were from dermatology, 76 from surgical, 74 from adult medical, 18 from pediatric and 29 from intensive care units. One hundred and sixty-six (58.4\%) isolates were from male patients, whereas 118 (41.5\%) were from females. The lowest and highest age at which S. aureus was isolated was from the pus sample of a 6-day-old male child and from the sputum sample of an 85-year-old male elderly patient, respectively. The underlying disorders of the patients are summarized in Table 1, with majority (65.8\%) of patients having skin and skin-structure infections.
Table 1: Underlying disorders of the patients from whom Staphylococcus aureus were isolated $(n=284)$

\begin{tabular}{|c|c|}
\hline Underlying disorder & Number of patients (\%) \\
\hline Skin and skin-structure infections ${ }^{a}$ & $187(65.8)$ \\
\hline Respiratory tract infection ${ }^{\mathrm{b}}$ & $41(14.4)$ \\
\hline Genitourinary tract disease ${ }^{c}$ & $18(6.3)$ \\
\hline Neurologic diseases $^{d}$ & $12(4.2)$ \\
\hline Malignancies ${ }^{e}$ & $12(4.2)$ \\
\hline Gastrointestinal pathology ${ }^{\dagger}$ & $10(3.5)$ \\
\hline Undetermined & $4(1.4)$ \\
\hline
\end{tabular}

ancludes chronic nonhealing wound ulcer (64 patients), subcutaneous abscess with or without discharging sinus (59), pemphigus vulgaris (22), breast abscess (13), postoperative wound infection (10), pyoderma (8), cervical lymphadenitis (5), furuncle (4), and erysipelas (2), 'Includes pneumonia (30 patients), chronic obstructive pulmonary disease (7), and pleural effusion (4), Includes acute urinary tract infection (13 patients), nephrotic syndrome (2), dysfunctional urinary bladder (2), and ureteric calculi (1), Includes brain abscess (10) and meningitis (2), elncludes carcinoma breast (4 patients), hematologic malignancy (4), carcinoma tongue (2), and carcinoma stomach (2), ${ }^{\prime}$ Chronic liver disease (9 patients) and mesenteric abscess (1)

A total of 127 (44.7\%) isolates were MRSA. The proportion of MRSA recovered from the OPD patients was $40.5 \%$ (32/79), whereas that from the admitted patients was $46.3 \%$ (95/205). Frequency of MRSA was highest in the respiratory samples $(21 / 31,67.7 \%)$, followed by urine $(16 / 27,59.2 \%)$, blood $(14 / 25,56.0 \%)$, pus $(71 / 175,40.5 \%)$, and catheter tips $(3 / 8,37.5 \%)$. As compared to the MSSA isolates, MRSA were significantly more resistant to almost all the conventional antimicrobials, except gentamicin, erythromycin, chloramphenicol, and nitrofurantoin [Table 2]. Two hundred and fifty-one (88.3\%) isolates were fully susceptible to vancomycin, whereas RVS (including VISA and hVISA) was observed in $33(11.6 \%)$ isolates [Table 2]. Overall, high resistance was observed to penicillin $(232 / 284,81.7 \%)$, erythromycin $(177 / 284$, $62.3 \%)$, ciprofloxacin $(148 / 284,52.1 \%)$, ofloxacin $(146 / 248,51.4 \%)$, clindamycin $(126 / 284,44.3 \%)$, trimethoprim-sulfamethoxazole (120/284, 42.2\%), and levofloxacin $(71 / 284,25.0 \%)$. Resistance was low to gentamicin $(15 / 284,5.3 \%)$, rifampicin $(23 / 284,8.1 \%)$, and doxycycline $(27 / 284,9.5 \%)$, whereas no resistance was observed to nitrofurantoin in the urinary isolates. Multidrug resistance was observed in 158 (55.6\%) isolates, and the D-test was positive in $65(22.8 \%)$ isolates [Table 2].

Table 3 shows the MIC distribution of MRSA and MSSA to vancomycin, which reveals that 266 (93.6\%) isolates were susceptible to vancomycin, whereas 18 (6.3\%, 9 MRSA and 9 MSSA) displayed intermediate resistance. Maximum isolates $(41.5 \%)$ demonstrated a vancomycin MIC of $2 \mu \mathrm{g} / \mathrm{ml}$, followed by $1.5 \mu \mathrm{g} / \mathrm{ml}$ (33.8\%). Among 18 VISA, 13 (72.2\%) had vancomycin MIC $3 \mu \mathrm{g} / \mathrm{ml}, 3(16.6 \%)$ had MIC $4 \mu \mathrm{g} / \mathrm{ml}$, and $2(11.1 \%)$ had vancomycin MIC $6 \mu \mathrm{g} / \mathrm{ml}$. Among the S. aureus strains with vancomycin MIC in the susceptible range ( $\leq 2 \mu \mathrm{g} / \mathrm{ml})$, hVISA phenotype was detected in 
$15(5.6 \%)$ of the 266 VSSA isolates. Thus, a total of 33 isolates $(11.6 \%, 18$ VISA and $13 \mathrm{hVISA})$ demonstrated RVS, whereas $251(88.3 \%)$ isolates demonstrated full susceptibility to vancomycin. The proportion of isolates with RVS was higher in MRSA $(17 / 127,13.3 \%)$ compared to MSSA (16/157, 10.1\%); however, this was not statistically significant. The sources of hVISA were pus, urine, respiratory samples, and blood (11, 2,1 , and 1 , respectively). There was a concordance of $97.8 \%$ between the results of agar dilution and Etest methods for vancomycin susceptibility. All the $S$. aureus isolates (both MRSA and MSSA) were uniformly susceptible to linezolid (MIC range,

Table 2: Susceptibility profile of Staphylococcus aureus isolates $(n=284)$ to various antimicrobials and to vancomycin

\begin{tabular}{|c|c|c|c|}
\hline \multirow[t]{2}{*}{$\begin{array}{l}\text { Antibiotic/or resistance } \\
\text { phenotype }\end{array}$} & \multicolumn{3}{|c|}{$\begin{array}{l}\text { Number of resistant isolates, } \\
\qquad n(\%)\end{array}$} \\
\hline & $\begin{array}{c}\text { MRSA } \\
(n=127) \\
\end{array}$ & $\begin{array}{l}\text { MSSA } \\
(n=157)\end{array}$ & $\begin{array}{c}\text { Total } \\
(n=284)\end{array}$ \\
\hline Penicillin & $127(100)^{*}$ & $105(66.8)$ & $232(81.7)$ \\
\hline Gentamicin & $9(7.1)$ & $6(3.8)$ & $15(5.3)$ \\
\hline Doxycycline & $18(14.1)^{*}$ & $9(5.7)$ & $27(9.5)$ \\
\hline Ciprofloxacin & $88(69.3)^{*}$ & $60(38.2)$ & $148(52.1)$ \\
\hline Levofloxacin & $47(37.0)^{*}$ & $24(15.3)$ & $71(25.0)$ \\
\hline Ofloxacin & $86(67.7)^{*}$ & $60(38.2)$ & $146(51.4)$ \\
\hline Erythromycin & $86(67.7)$ & $91(57.9)$ & $177(62.3)$ \\
\hline Clindamycin & $66(51.9)^{*}$ & $60(38.2)$ & $126(44.3)$ \\
\hline Trimethoprim-sulfamethoxazole & $74(58.2)^{*}$ & $46(29.2)$ & $120(42.2)$ \\
\hline Chloramphenicol & $20(15.7)$ & $29(18.4)$ & 49 (17.2) \\
\hline Rifampicin & $21(16.5)^{*}$ & $2(1.3)$ & $23(8.1)$ \\
\hline Nitrofurantoin ${ }^{\#}$ & 0 & 0 & 0 \\
\hline Linezolid & 0 & 0 & 0 \\
\hline Daptomycin & 0 & 0 & 0 \\
\hline Tigecycline & 0 & 0 & 0 \\
\hline D-test positive & $36(28.3)$ & $29(18.4)$ & $65(22.8)$ \\
\hline Multidrug resistance & $127(100)^{*}$ & 67 (42.6) & $158(55.6)$ \\
\hline \multicolumn{4}{|l|}{ Vancomycin } \\
\hline $\mathrm{MIC}$ range $(\mu \mathrm{g} / \mathrm{ml})$ & $1-4$ & $0.75-6$ & $0.75-6$ \\
\hline $\mathrm{MIC}_{50}(\mu \mathrm{g} / \mathrm{ml})$ & 2 & 2 & 2 \\
\hline $\mathrm{MIC}_{90}(\mu \mathrm{g} / \mathrm{ml})$ & 2 & 2 & 2 \\
\hline VISA & $9(7.1)$ & $9(5.7)$ & $18(6.3)$ \\
\hline hVISA & $8(6.3)$ & $7(4.5)$ & $15(5.3)$ \\
\hline RVS & $17(13.3)$ & $16(10.1)$ & $33(11.6)$ \\
\hline
\end{tabular}

${ }^{*} P<0.05$ (significant) for difference between MRSA and MSSA by Chi-square test, "Tested only in urinary isolates. S. aureus=Staphylococcus aureus, MRSA=Methicillin-resistant $S$. aureus, MSSA=Methicillin-susceptible $S$. aureus, $\mathrm{MIC}=$ Minimum inhibitory concentration, $\mathrm{VISA}=$ Vancomycin-intermediate $S$. aureus, hVISA=heterogeneous VISA; RVS=Reduced vancomycin susceptibility
$1.5-2 \mu \mathrm{g} / \mathrm{ml}$ ), tigecycline (MIC range, $0.064-0.094 \mu \mathrm{g} /$ $\mathrm{ml}$ ), and daptomycin (MIC range, $0.19-0.38 \mu \mathrm{g} / \mathrm{ml}$ ).

\section{Discussion}

The study presents a comprehensive data of MRSA and MSSA in a tertiary care hospital in the Eastern part of India, which receives patients not only from the local areas on a primary basis but also large referrals from adjoining districts and states. The study isolates comprised mainly skin and soft tissue $(61.6 \%)$, followed by respiratory (10.9\%) and urine $(9.5 \%)$ isolates, as has been found in previous observational studies, ${ }^{[25,26]}$ where $S$. aureus was mainly isolated from skin and soft-tissue infections (52.5\%-64\%), followed by other samples in varying frequency. The study also revealed a prevalence of $44.7 \%$ (127 out of 284) MRSA among the $S$. aureus isolates, which is comparable to previously reported studies from India in recent times, with a prevalence of $38.4 \%-54.8 \% .{ }^{[25-27]}$ Further, among 127 MRSA isolates, 91 (71.6\%) were MDR, which is similar to the study by Tiwari et al., ${ }^{[27]}$ where among 783 isolates of S. aureus, 301 (38.4\%) were methicillin resistant, of which 217 (72.1\%) were found to be MDR. Similar to previous studies, ${ }^{[12,25,26]}$ the present study also observed that MRSA isolates were significantly more resistant to various antibiotics, such as penicillin, trimethoprim-sulfamethoxazole, fluoroquinolones, erythromycin, and clindamycin as well as they exhibited significantly more multidrug resistance.

As regards vancomycin susceptibility, hVISA was first reported in Japan in 1997 and along with VISA has since been identified worldwide from many countries including the United States, Japan, Australia, France, Scotland, Brazil, South Korea, Hong Kong, South Africa, Thailand, Israel, and others. ${ }^{[3,4]}$ In India, Menezes et al. ${ }^{[15]}$ in 2008 reported the emergence of vancomycin-intermediate Staphylococcus species in Southern India using the revised guidelines. Of 102 oxacillin-resistant $S$. aureus isolates, one was found to be a VISA strain (MIC $5 \mu \mathrm{g} / \mathrm{ml}) \cdot{ }^{[15]} \mathrm{In}$ a study from Western region of India, among 58 clinical isolates of MRSA, the prevalence of hVISA was observed to be $6.9 \%$ with PAP-AUC and macromethod Etest. ${ }^{[16]}$ In a study from South India (Hyderabad) performed on 358 S. aureus isolates only from intensive care units, $285(79.6 \%)$ were identified as MRSA. ${ }^{[28]}$ Sixteen isolates

Table 3: Minimum inhibitory concentration distribution of methicillin-resistant Staphylococcus aureus and methicillin-susceptible Staphylococcus aureus to vancomycin

\begin{tabular}{lcccccccc}
\hline S. aureus isolates & \multicolumn{7}{c}{ MIC to vancomycin $(\mu \mathrm{g} / \mathrm{ml})$ by Etest } \\
\cline { 2 - 8 } & 0.75 & $\mathbf{1}$ & 1.5 & 2 & 3 & 4 & 6 \\
\hline MRSA & 0 & 23 & 44 & 51 & 7 & 2 & 0 \\
MSSA & 2 & 27 & 52 & 67 & 6 & 1 & 2 & 127 \\
Total (\%) & $2(0.7)$ & $50(17.6)$ & $96(33.8)$ & $118(41.5)$ & $13(4.5)$ & $3(1.1)$ & $2(0.7)$ & 284 \\
\hline
\end{tabular}

S. aureus=Staphylococcus aureus, MRSA=Methicillin-resistant $S$. aureus, MSSA=Methicillin-susceptible $S$. aureus, MIC=Minimum inhibitory concentration 
showed an MIC range between 4 and $8 \mu \mathrm{g} / \mathrm{ml}$, indicating vancomycin-intermediate resistance, and seven isolates showed an MIC range between 16 and $64 \mu \mathrm{g} / \mathrm{ml}$, indicating VRSA. ${ }^{[28]}$ PCR amplification for van $A$ among the seven VRSA showed that six contained van $A$ and one was negative for van $A .{ }^{[28]}$ Apart from these, two studies from North India have observed the presence of VISA and hVISA in their setup. ${ }^{[18,19]}$ In Karachi (a neighboring region of India), of 450 S. aureus isolates, 174 (38.6\%) were found to be MRSA. ${ }^{[29]}$ One isolate was found to be vancomycin resistant (MIC $32 \mu \mathrm{g} / \mathrm{ml}$ ), whereas four isolates had intermediate resistance, with two strains having MIC of $16 \mu \mathrm{g} / \mathrm{ml}$ and two having MIC of $8 \mu \mathrm{g} / \mathrm{ml} .{ }^{[29]}$ Thus, the hVISA and VISA rate found in the current study is similar to the previously reported studies in India and demonstrates the emergence of RVS in yet another region (Eastern) of India. Further, though the hVISA rate found in the current study appears low $(6.3 \%$ of MRSA and $4.5 \%$ of MSSA), the total rate of RVS (hVISA and VISA) is moderately high ( $11.6 \%$ overall, $13.3 \%$ in MRSA, and $10.1 \%$ in MSSA). Apart from MRSA, several studies have found RVS in MSSA isolates, which has been observed to be associated with poorer outcomes and increased complications. ${ }^{[5,7,8]}$ One study has shown that the development of intermediate vancomycin susceptibility in MSSA may result in increased tolerance to several other classes of antistaphylococcal antibiotics. ${ }^{[30]}$ The clinical implications of RVS observed in MSSA isolates in our setup needs to be investigated further by prospective cohort studies.

\section{Conclusion}

The present study shows that a moderately high percentage of $S$. aureus demonstrated RVS, which may limit its usefulness in MRSA and may be associated with increased complications in MSSA infections. Linezolid, tigecycline, daptomycin, and doxycycline may be considered as alternatives for the treatment of isolates with reduced susceptibility to vancomycin. In view of increasing glycopeptide resistance, the susceptibility status of vancomycin along with other antibiotics among clinical S. aureus isolates should be investigated periodically.

\section{Financial support and sponsorship \\ Nil.}

\section{Conflicts of interest}

There are no conflicts of interest.

\section{References}

1. Boucher HW, Corey GR. Epidemiology of methicillin-resistant Staphylococcus aureus. Clin Infect Dis 2008;46 Suppl 5:S344-9.

2. Valle DL Jr., Paclibare PA, Cabrera EC, Rivera WL. Molecular and phenotypic characterization of methicillin-resistant Staphylococcus aureus isolates from a tertiary hospital in the Philippines. Trop Med Health 2016;44:3.

3. Koh YR, Kim KH, Chang CL, Yi J. Prevalence and clinical impact of heterogeneous vancomycin-intermediate Staphylococcus aureus isolated from hospitalized patients. Ann Lab Med 2016;36:235-43.

4. Howden BP, Davies JK, Johnson PD, Stinear TP, Grayson ML. Reduced vancomycin susceptibility in Staphylococcus aureus, including vancomycin-intermediate and heterogeneous vancomycin-intermediate strains: Resistance mechanisms, laboratory detection, and clinical implications. Clin Microbiol Rev 2010;23:99-139.

5. Hu J, Ma XX, Tian Y, Pang L, Cui LZ, Shang H. Reduced vancomycin susceptibility found in methicillin-resistant and methicillin-sensitive Staphylococcus aureus clinical isolates in Northeast China. PLoS One 2013;8:e73300.

6. Lin SY, Chen TC, Chen FJ, Chen YH, Lin YI, Siu LK, et al. Molecular epidemiology and clinical characteristics of hetero-resistant vancomycin intermediate Staphylococcus aureus bacteremia in a Taiwan medical center. J Microbiol Immunol Infect 2012;45:435-41.

7. Aguado JM, San-Juan R, Lalueza A, Sanz F, Rodríguez-Otero J, Gómez-Gonzalez C, et al. High vancomycin MIC and complicated methicillin-susceptible Staphylococcus aureus bacteremia. Emerg Infect Dis 2011;17:1099-102.

8. San-Juan R, Viedma E, Chaves F, Lalueza A, Fortún J, Loza E, et al. High MICs for vancomycin and daptomycin and complicated catheter-related bloodstream infections with methicillin-sensitive Staphylococcus aureus. Emerg Infect Dis 2016;22:1057-66.

9. Clinical and Laboratory Standards Institute. Performance Standards for Antimicrobial Susceptibility Testing: $16^{\text {th }}$ Informational Supplement. Clinical and Laboratory Standards Institute Document M100-S16. Wayne, PA: Clinical and Laboratory Standards Institute; 2006.

10. Clinical and Laboratory Standards Institute. Performance Standards for Antimicrobial Susceptibility Testing: 19 ${ }^{\text {th }}$ Informational Supplement. Clinical and Laboratory Standards Institute Document M100-S19. 19 $9^{\text {th }}$ ed.. Wayne, PA: Clinical and Laboratory Standards Institute; 2009.

11. Leonard SN, Rossi KL, Newton KL, Rybak MJ. Evaluation of the etest GRD for the detection of Staphylococcus aureus with reduced susceptibility to glycopeptides. J Antimicrob Chemother 2009;63:489-92.

12. Di Gregorio S, Perazzi B, Ordoñez AM, De Gregorio S, Foccoli M, Lasala MB, et al. Clinical, microbiological, and genetic characteristics of heteroresistant vancomycin-intermediate Staphylococcus aureus bacteremia in a teaching hospital. Microb Drug Resist 2015;21:25-34.

13. Holubar M, Meng L, Deresinski S. Bacteremia due to methicillin-resistant Staphylococcus aureus: New therapeutic approaches. Infect Dis Clin North Am 2016;30:491-507.

14. Culos KA, Cannon JP, Grim SA. Alternative agents to vancomycin for the treatment of methicillin-resistant Staphylococcus aureus infections. Am J Ther 2013;20:200-12.

15. Menezes GA, Harish BN, Sujatha S, Vinothini K, Parija SC. Emergence of vancomycin-intermediate Staphylococcus species in Southern India. J Med Microbiol 2008;57:911-2.

16. Chaudhari CN, Tandel K, Grover N, Sen S, Bhatt P, Sahni AK, et al. Heterogeneous vancomycin-intermediate among methicillin resistant Staphylococcus aureus. Med J Armed Forces India 2015;71:15-8.

17. Bakthavatchalam YD, Veeraraghavan B, Peter JV, Rajinikanth J, Inbanathan FY, Devanga Ragupathi NK, et al. Novel observations in 11 heteroresistant vancomycin-intermediate methicillin-resistant Staphylococcus aureus strains from South India. Genome Announc 2016;4. pii: e01425-16.

18. Jain S, Gaind R, Chugh TD. In vitro activity of vancomycin and daptomycin against clinical isolates of Staphylococcus aureus and 
enterococci from India. Int J Antimicrob Agents 2013;42:94-5.

19. Singh A, Prasad KN, Misra R, Rahman M, Singh SK, Rai RP, et al. Increasing trend of heterogeneous vancomycin intermediate Staphylococcus aureus in a tertiary care center of Northern India. Microb Drug Resist 2015;21:545-50.

20. Collee JG, Miles RS, Watt B. Tests for the identification of bacteria. In: Collee JG, Fraser AG, Marmion BP, Simmons A, editors. Mackie and McCartney Practical Medical Microbiology. $14^{\text {th }}$ ed. London: Churchill Livingstone; 1996. p. 131-45.

21. Clinical and Laboratory Standards Institute. Performance Standards for antimicrobial Susceptibility Testing: $25^{\text {th }}$ Informational Supplement. Clinical and Laboratory Standards Institute Document M100-S25. 25 $5^{\text {th }}$ ed.. Wayne, PA: Clinical and Laboratory Standards Institute; 2015.

22. Holmes NE, Johnson PD, Howden BP. Relationship between vancomycin-resistant Staphylococcus aureus, vancomycin-intermediate $S$. aureus, high vancomycin MIC, and outcome in serious S. aureus infections. J Clin Microbiol 2012;50:2548-52.

23. Pfizer Inc. (Wyeth Pharmaceuticals): Tygacil ${ }^{\circledR}$ Product Insert. Pfizer; 2013. Available from: http://www.pfizerpro.com/hcp/ tygacil. [Last accessed on $2016 \mathrm{Jul} 15]$.

24. Magiorakos AP, Srinivasan A, Carey RB, Carmeli Y, Falagas ME, Giske CG, et al. Multidrug-resistant, extensively drug-resistant and pandrug-resistant bacteria: An international expert proposal for interim standard definitions for acquired resistance. Clin Microbiol Infect 2012;18:268-81.

25. Indian Network for Surveillance of Antimicrobial Resistance (INSAR) group, India. Methicillin resistant Staphylococcus aureus (MRSA) in India: Prevalence and susceptibility pattern. Indian J Med Res 2013;137:363-9.

26. Anupurba S, Sen MR, Nath G, Sharma BM, Gulati AK, Mohapatra TM. Prevalence of methicillin resistant Staphylococcus aureus in a tertiary referral hospital in Eastern Uttar Pradesh. Indian J Med Microbiol 2003;21:49-51.

27. Tiwari HK, Sapkota D, Sen MR. High prevalence of multidrug-resistant MRSA in a tertiary care hospital of Northern India. Infect Drug Resist 2008;1:57-61.

28. Thati V, Shivannavar CT, Gaddad SM. Vancomycin resistance among methicillin resistant Staphylococcus aureus isolates from intensive care units of tertiary care hospitals in Hyderabad. Indian J Med Res 2011;134:704-8.

29. Taj Y, Abdullah FE, Kazmi SU. Current pattern of antibiotic resistance in Staphylococcus aureus clinical isolates and the emergence of vancomycin resistance. J Coll Physicians Surg Pak 2010;20:728-32.

30. Pillai SK, Wennersten C, Venkataraman L, Eliopoulos GM, Moellering RC, Karchmer AW. Development of reduced vancomycin susceptibility in methicillin-susceptible Staphylococcus aureus. Clin Infect Dis 2009;49:1169-74. 